\title{
TRAIL Enhances Shikonin Induced Apoptosis through ROS/JNK Signaling in Cholangiocarcinoma Cells
}

\author{
Guangyao Zhou ${ }^{\mathrm{a}, \mathrm{b}}$ Zuqin Yang ${ }^{\mathrm{c}}$ Xiaodong Wanga,d Ran Tao ${ }^{\mathrm{e}}$ Yuanping Zhou ${ }^{\mathrm{a}}$ \\ aDepartment of Infectious Diseases, Nanfang Hospital, Southern Medical University, Guangzhou, \\ bDepartment of Infectious Diseases, the Second Affiliated Hospital \& Yuying Children's Hospital of \\ Wenzhou Medical University, Wenzhou, 'Department of Pediatrics, the Second Affiliated Hospital \\ \& Yuying Children's Hospital of Wenzhou Medical University, Wenzhou, dDepartment of Infectious \\ Diseases, the First Affiliated Hospital of Wenzhou Medical University, Wenzhou, ${ }^{e}$ Center for Clinical \\ Medical Research, Zhejiang Provincial People's Hospital, Hangzhou, PR China
}

\section{Key Words}

Shikonin $•$ TRAIL $•$ Cholangiocarcinoma $•$ Apoptosis $・$ ROS/JNK $・$ DR5

\begin{abstract}
Background/Aims: Cholangiocarcinoma (CCA), arising from varying locations within the biliary tree, is the second most common primary liver malignancy worldwide. Shikonin, an active compound extracted from the Chinese herb Zicao, holds anti-bacterial, anti-inflammatory, and anti-tumor activities. However, the effect of shikonin on human cholangiocarcinoma and detailed mechanisms of TRAIL enhancement remains to be elucidated. The purpose of the study was to investigate the protective functions of TRAIL enhancement for shikonin induced apoptosis in cholangiocarcinoma cells. Methods: We use MTT assay, apoptosis assay, caspase activity assay, flow cytometry assay, real time PCR and Western blot to observe the effects of TRAIL on shikonin induced cholangiocarcinoma cells apoptosis and its mechanism. Results: Shikonin inhibited cell viability and induced apoptosis of CCA cells, effects enhanced by TRAIL treatment via activation of caspase-3, -8, -9. Furhermore, TRAIL enhanced anti-proliferation of shikonin and shikonin induced apoptosis through induction of ROS mediated JNK activation, while AKT activation had an effect on shikonin anti-proliferation activity, but not in the TRAIL enhanced counterparts. Finally, shikonin upregulated DR5 expression, an effect essential for TRAIL-enhanced activities of shikonin in RBE cells. Conclusions: Our results revealed that shikonin could inhibit cells viability and induce apoptosis of CCA cells, effects enhanced by TRAIL treatment via ROS mediated JNK signalling pathways, involving up-regulation of DR5 expression. Our results provide further insight into the mechanism underlying the anti-tumor effects of shikonin by TRAIL enhanced in CCA and a new therapeutic strategy to CCA treatment.
\end{abstract}

Professor Yuanping Zhou and Ran Tao, MD, FACS,

Professor of Surgery
State Key Lab Organ Fail Res, Guangdong Provincial Key Lab Viral Hepatitis Res, Department of Infectious Diseases, Nanfang Hospital, Southern Medical University, 1838 North Guangzhou Avenue, Guangzhou, Guangdong 510515, (P.R. China); Dept Hepatobil-Pancreatic Surg, Zhejiang Provincial People's Hospital (ZJPPH). 14th Floor Building 1, 158 Shangtang Road, Hangzhou, Zhejiang Province, 310014, (PR China) E-Mail zhouyuanping15@163.com / taoran898@163.com 


\section{Cellular Physiology Cell Physiol Biochem 2017;42:1073-1086 \begin{tabular}{l|l} 
DOI: 10.1159/000478758 \\
and Biochemistry
\end{tabular} \begin{tabular}{l}
$\begin{array}{l}\text { O 2017 The Author(s). Published by S. Karger AG, Basel } \\
\text { www.karger.com/cpb }\end{array}$ \\
\cline { 2 - 3 }
\end{tabular}}

Zhou et al.: Mechanism Role of Shikonin in Cholangiocarcinoma

\section{Introduction}

Cholangiocarcinoma (CCA) is a malignant tumor of bile duct epithelial cells and the second most common primary liver malignancy, accounting for $10 \% \sim 15 \%$ of all primary liver cancers [1]. CCA arises from varying locations within the biliary tree, classified as intrahepatic, perihilar or extrahepatic CCA [2]. The development of CCA is a multi-step process, and may be induced by sclerosing cholangitis, hepatolithiasis, parasite infections and toxins $[3,4]$. The clinical presentation of CCA patients is rather unspecific. Biologically, CCA is one of the highly aggressive tumors with dismal prognosis. The incidence and mortality of CCA have been increasing across the world during recent years [5]. In the last two decades, treatment options of CCA have been improved. Several molecules and signaling pathways have been developed as the therapeutic targets that interfere with growth and progression of cancer cells while sparing normal cells, such as IL-6, fibroblast growth factor receptor (FGFR), notch signaling pathway, receptor tyrosine kinase pathway and PI3K/AKT/ mTOR pathway [6]. However, new therapeutic strategies are still urgently needed to improve the treatment outcome of CCA.

Shikonin, a well-known traditional Chinese medicine from the root extract of Lithospermum erythrorhizon Sieb. et Zucc. (Boraginaceae), is one of the active constituents of Zicao. Shikonin was well illustrated for its anti-inflammatory effect in animal models [7]. Pre-treatment with shikonin significantly suppressed the carrageenan-induced paw edema and the development of chronic arthritis [8]. The anti-inflammatory role of shikonin may contribute to its therapeutic effect in cancer, since chronic inflammation promotes the initiation and progression of cancers. Recently numerous studies have illustrated that shikonin held anti-cancer potential in various cancers, including lung, colon, breast, osteosarcoma cancer and acute myeloid leukemia [9-13]. Shikonin inhibited the metastasis of cancer cells by suppressing matrix metalloproteinase-2/-9 expression as well as matrix metalloproteinase-13 [14-16]. LPS-induced epithelial mesenchymal transition (EMT) was reduced by shikonin via inactivation of NF- $\kappa B$ signaling pathway [17]. Shikonin also induced the apoptosis of cancer cells through the dual induction of the endoplasmic reticulum (ER) stress and the mitochondrial dysfunction $[18,19]$. The up-regulation of p73 and downregulation of ICBP90 during the shikonin treatment also led to the apoptosis of human cancer cells [20]. Shikonin could induce apoptosis, necrosis by up-regulating p53 and Nrf2 expression in lung cancer and stomach carcinoma [21,22]. Therefore, to study the effect of shikonin on the CCA cells is significant for the new method of clinical therapy in CCA.

Tumor necrosis factor-related apoptosis-inducing ligand (TRAIL) was well known for its function of inducing cell death in various cancer cells [23]. Previously reported that TRAIL could inhibit cell proliferation and induce apoptosis in CCA cells by combination with subtoxic 5-FU [24]. However, there was a major barrier that low TRAIL receptor expression levels in CCA cells led to resistance to TRAIL. Moreover, previous studies revealed that triptolide sensitized resistant CCA Cells to TRAIL-induced apoptosis [25]. DR5 was a cell death receptor involved in the regulation of TRAIL induced apoptosis. Zhong et al. found guggulsterone could increase DR5 protein expression level in a dose dependent manner in CCA cells [26]. However, the effects of shikonin on DR5 expression level and combination of TRAIL and shikonin on apoptosis of CCA cells remains to be further investigated.

Multiple target molecules and signaling pathways have been involved in the antitumor role of shikonin in human cancers, but the detailed underlying mechanism remains obscure, including apoptosis, necrosis, and anti-angiogenesis. Whether shikonin has an antitumor effect on CCA cells and the effects of TRAIL on shiknin activity for CCA cells, and the molecular mechanism involved remain unknown. Here, we found that shikonin could inhibit cell viability and induce apoptosis of CCA cells, effects enhanced by TRAIL treatment via activation of caspase-3, -8, -9. As further investigation demonstrated, TRAIL enhanced antiproliferation of shikonin and shikonin induced apoptosis through induction of ROS mediated JNK activation, while AKT activation had an effect on shikonin anti-proliferation activity, but 


\section{Cellular Physiology Cell Physiol Biochem 2017;42:1073-1086 \begin{tabular}{l|l} 
DOI: 10.1159/000478758 & $\begin{array}{l}\text { O 2017 The Author(s). Published by S. Karger AG, Basel } \\
\text { www.karger.com/cpb }\end{array}$ \\
\hline
\end{tabular}}

Zhou et al.: Mechanism Role of Shikonin in Cholangiocarcinoma

not in the TRAIL enhanced counterparts. Finally, we also found shikonin upregulated DR5 expression and its effect was essential for TRAIL-enhanced effects of shikonin in RBE cells.

\section{Materials and Methods}

Medicines and antibodies

Shikonin was obtained from Enzo Life Sciences (Farmingdale, NY, USA). Q-VD-OPh was purchased from R\&D Systems (Minneapolis, MN, USA); SP600125, SB202190 and N-acetyl-L-cysteine (NAC) were purchased from Sigma (St. Louis, MO, USA), and NAC was dissolved in water. LY294002 was obtained from Cell Signaling Technology (Beverly, MA, USA) Catalase was obtained from Sigma (St. Louis, MO, USA) and dissolved in potassium phosphate buffer.

\section{Cell culture}

RBE cholangiocarcinoma cell lines, were obtained from Cell Bank of CAS (Chinese Academy of Sciences) (Shanghai, China). RPMI-1640 medium supplemented with $100 \mathrm{U} / \mathrm{mL}$ penicillin, $100 \mu \mathrm{g} / \mathrm{mL}$ streptomycin and $10 \%$ fetal bovine serum was used to maintain these cells in a humidified incubator at $37^{\circ} \mathrm{C}$ in an atmosphere of $5 \% \mathrm{CO}_{2}$.

\section{Cell viability assay}

Cell viability was determined by the MTT assay. After treatment, 3- (4, 5-dimethylthiazol-2-yl) 2, 5-diphenylte-trazolium bromide (MTT) solution was added and incubated for 4 hours. After removing of the medium, $100 \mathrm{~mL}$ DMSO was added and the absorbance was measured at $490 \mathrm{~nm}$ with the FlexStation microplate reader (Silicon Valley, CA, USA).

\section{Apoptosis Assay}

Apoptosis assay was performed using the apoptosis detection kit (Biolegend, CA, USA) according to the manufacturer's instructions. RBE cells were treated with the indicated reagents after a certain period of time, and stained with $5 \mu \mathrm{g} / \mathrm{ml}$ 7-aminoactinomycin D (7-AAD) and $2.5 \mu \mathrm{g} / \mathrm{ml}$ Annexin V-fluorescein isothiocyanate (FITC), and analyzed by flow cytometry on a FACSCalibur (BD Biosciences, CA, USA).

\section{Real-time PCR}

Total RNA was extracted using the TRIzol reagent (Invitrogen, Carlsbad, USA). Reverse-transcribed complementary DNA was synthesized with the Prime-Script® RT Reagent Kit (TaKaRa, Tokyo, Japan). Primers for DR5 and GAPDH were purchased from Sangon Biotech (Shanghai) Co.,Ltd. DR5 forward primer (5'-AGACCCTTGTGCTCGTTGTC-3'); reverse primer (5'-TTGTTGGGTGATCAGAGCAG-3'). GAPDH: forward primer (5'-AATC CCATCACCATCTTCCA-3'); reverse primer (5'-TGGACTCCACGACGTACTCA-3'). PCR conditions for the reactions were as follows: $95^{\circ} \mathrm{C}$ for $10 \mathrm{~s}, 58^{\circ} \mathrm{C}$ for $30 \mathrm{~s}$ and $72^{\circ} \mathrm{C} 10 \mathrm{~s}$ for 40 cycles. The PCR products were done using a Power SYBR Green kit and performed with LightCycler 480 (Roche, Welwyn Garden, Switzerland). The calculated amounts of DR5 mRNAs were normalized to GAPDH. The results were presented as the ratio of cycle threshold (Ct) value for GAPDH.

\section{Western blot}

Protein extracted from cells was treated with 10\% sodium dodecyl sulfate-polyacrylamide gel (SDSPAGE). Then the gel was transferred on to a nitrocellulose membrane (Bio-Rad, Hercules, USA). and the membrane was incubated with 5\% non-fat milk for 2 hours. Rabbit or mouse primary antibodies were hybridized the membrane. The primary antibodies were used as follows: cleaved caspase-3, 8, 9, phosphoJNK, phospho-ERK1/2, phospho-p38, DR-5, phospho-Akt and GAPDH antibodies were obtained from Cell Signaling Technology (Beverly, MA, USA). Bid, Bax and JBD antibodies were purchased from Santa Cruz Biotechnology (Santa Cruz, CA, USA).

\section{Measurement of caspase activity}

Caspase-3, Caspase-8 and Caspase-9 Colorimetric Assay Kits (Biovision, CA, USA) were utilized to detect the Caspase-3, -8 and -9 activities, respectively. The use of kits is in accordance with the manufacturer's protocols. 


\section{Cellular Physiology Cell Physiol Biochem 2017;42:1073-1086 \begin{tabular}{l|l} 
DOI: 10.1159/000478758 & $\begin{array}{l}\text { O 2017 The Author(s). Published by S. Karger AG, Basel } \\
\text { www.karger.com/cpb }\end{array}$ \\
\hline
\end{tabular}}

Zhou et al.: Mechanism Role of Shikonin in Cholangiocarcinoma

\section{Determination of intracellular reactive oxygen species (ROS) production}

An oxidationsensitive fluorescent probe, 2',7'-dichlorofluorescin diacetate (DCFH-DA, D6665; SigmaAldrich, St. Louis, MO) method was utilized to measure the formation of reactive oxygen species ground on the ROS-dependent oxidation of DCFH-DA to 2'7'-dichlorofluorescein (DCF) [27]. About $1 \times 10^{6} /$ well RBE cells plated on cover glasses in 12-well plates were grown for $72 \mathrm{~h}$. The cells were treated with $0,1,2,5$ or $10 \mu \mathrm{M}$ shikonin at $37^{\circ} \mathrm{C}$ for 2 hours. Removing the medium and washing by PBS. Then, incubating with 200 $\mu \mathrm{l}$ DCFH-DA $(10 \mu \mathrm{M})$ at $37^{\circ} \mathrm{C}$ in the dark for $30 \mathrm{~min}$. Intracellular ROS formation was measured using DCFHDA by the method previously described for the DCF-DA microplate assay [28]. A spectrofluorometer (SFM 25; Kontron Instruments) was used to measure ROS production by the fluorescence intensity of 10,000 cells in each well at an excitation wavelength of $495 \mathrm{~nm}$ and an emission wavelength of $530 \mathrm{~nm}$. Then the fluorescence intensity of the shikonin-stimulated cultures to set $100 \%$ after subtracting the corresponding blanks to calculate relative intensities of DCF fluorescence were calculated by setting.

\section{Measurement of catalase activity}

The level of catalase activity was measured using a catalase assay kit (Sigma-Aldrich, St. Louis, MO). In brief, $1 \times 10^{6}$ cells were washed and suspended in lysis buffer. The concentration of supernatant protein was determined by the Bradford method and. Supernatant samples were used to measure catalase activity. Endpoint absorbance was measured at $520 \mathrm{~nm}$ using a microplate reader (Spectra MAX, Molecular Devices Co.).

Analysis of DR5 cell surface expression

Cells were treated with indicated concentration of shikonin for $24 \mathrm{~h}, 1 \times 10^{5}$ cells were collected to the tube, DR5 monoclonal antibody (R\&D Systems) was incubated for $1 \mathrm{~h}$ at $4^{\circ} \mathrm{C}$ after washing and resuspending. Cells were washed and resuspended in PBS containing $0.5 \%$ BSA, and analyzed by flow cytometry on a FACSCalibur (BD Biosciences, CA, USA).

Adenovirus and plasmid vector construction, infection and transfection

HEK-293T cells were plated in culture dish. After HEK-293T cells attached to the plate overnight, the linearized DNA, the shuttle vector and pacAd vector were cotransfected into HEK-293T by Lipofectamine 2000 (Invitrogen, CA, USA). Viral lysates were harvested, purified and tittered. One day prior to infection, cells were seeded in plate. The next day, adenoviruses containing JBD (JNK binding domain) or AKT were added into the medium and infecting the cells. Oligonucleotides of small interfering RNA were ordered from Sangon Biotech (Shanghai) Co.,Ltd. The following siRNA was listed: DR5 siRNA 5'-AAGUUGCAGCCGUAGUCUUGA-3',

Statistical analysis

Results are presented as the means \pm S.D. from at least 3 independent experiments. The statistical differences were calculated by the Student's $t$-test or one-way analysis of variance (ANOVA) with Dunnett's test.

\section{Results}

TRAIL enhanced shikonin anti-proliferation activity and induced cell apoptosis in RBE cells

The anti-proliferation effect of shikonin was determined by MTT. We found the antiproliferation to RBE cells was increased with the high concentration of shikonin treatment (Fig. 1b). However, pretreatment with TRAIL could significantly enhanced shikonin induced cytotoxicity in RBE cells (Fig. 1c). We also investigated the effect of TRAIL on the shikonin induced apoptosis based on the observation of relative changes of annexin V-FITC and 7AAD, two well-known characteristics of apoptotic cell death. The percentage of apoptotic RBE cells was significantly increased in the group of RBE cells treated with TRAIL and shikonin, compared with TRAIL or shikonin alone (Fig. $1 \mathrm{f}$ and g). We further conducted western blot analysis to investigate the activation of apoptotic proteins including caspase-3,-8 and -9. As demonstrated in Fig. 1d, the protein expressions of caspase-3, -8 and -9 and BAX were obvious 
a

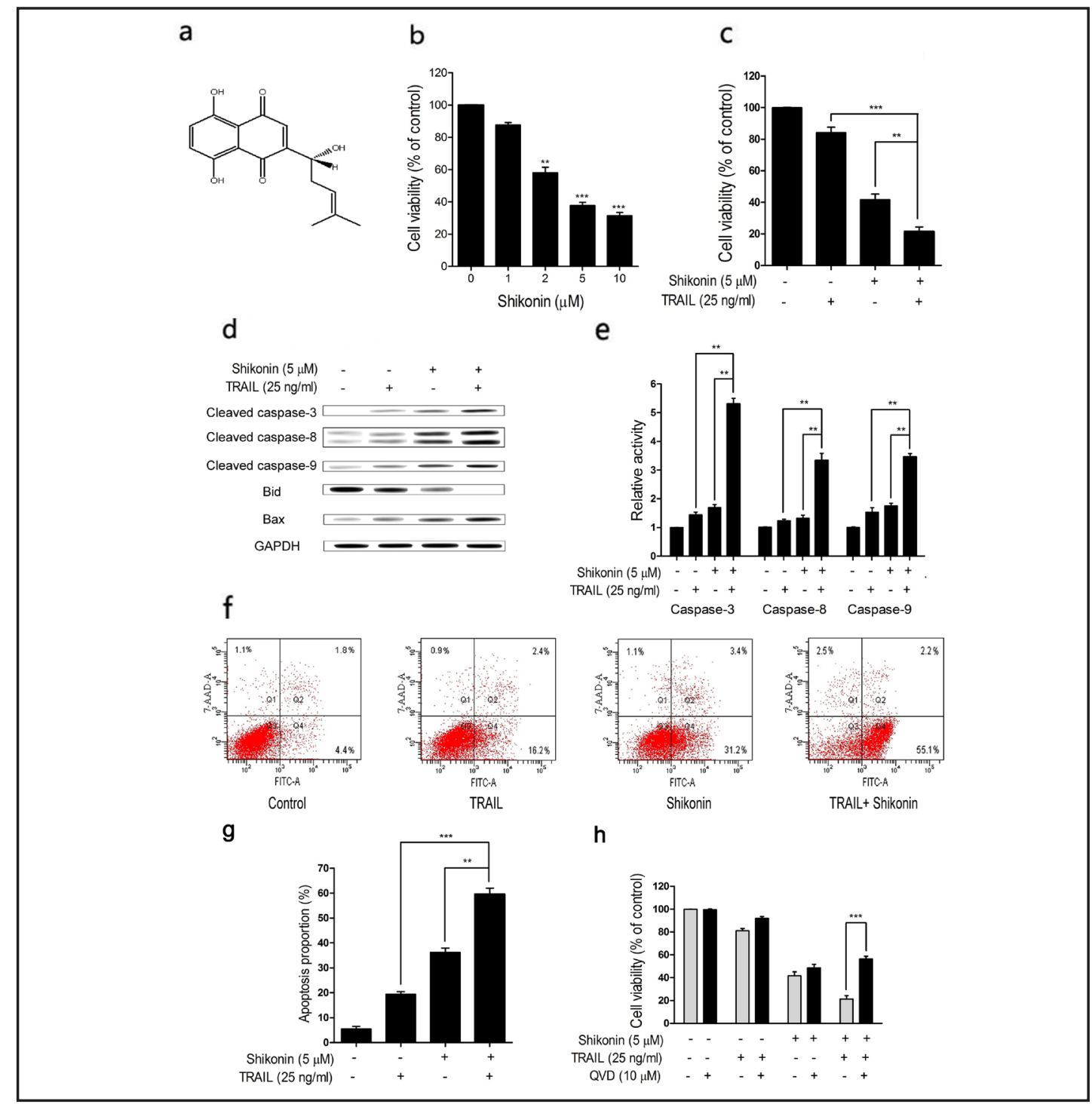

Fig. 1. TRAIL enhances shikonin inhibited cell proliferation and induced cell apoptosis in RBE CCA cells. (a) The structure of shikonin. (b) The anti-proliferative effect of shikonin on RBE cells was determined by MTT. (c) Cell viability was assessed after RBE cells were pretreated with TRAIL for $8 \mathrm{~h}$ followed by treatment with shikonin for $24 \mathrm{~h}$ (d) Western blotting analysis for protein expressions of Cleaved caspase-3, 8, 9, Bid and Bax were measured in RBE cells treated with shikonin after pretreatment with TRAIL for $8 \mathrm{~h}$. (e) Caspase activities in RBE cells that had been treated with TRAIL for $8 \mathrm{~h}$ and then shkonin for $24 \mathrm{~h}$. (f, g) Cells were treated with shikonin and/or TRAIL. Cells were stained with annexin V-FITC/7-AAD and analyzed by flow cytometry. (h) Cell viability was shown for RBE cells treated with TRAIL and/or shkonin followed by the pretreatment of pancaspase inhibitor Q-VD-OPh for $1 \mathrm{~h}$. Results are presented as the means \pm S.D. from at least 3 independent experiments. The statistical differences were calculated by the Student's t-test or oneway analysis of variance (ANOVA) with Dunnett's test. ${ }^{* *} \mathrm{P}<0.01,{ }^{* * *} \mathrm{P}<0.001$.

elevated in the group of RBE cells treated with TRAIL and shikonin. To further confirm their functional involvement of caspases, we evaluated their activities in the cell lysates. Exposure of RBE cells to shikonin and TRAIL treatment led to a dramaticlly increased activation of caspase--3, -8 and -9 (Fig. 1e). Bid protein (22 kDa) was a well-known downstream of caspase cascade that would be degraded by active caspase- 8 to generate the truncated Bid (tBid, 15 $\mathrm{kDa}$ ) and cause mitochondrial membrane transition pore opening with subsequent release 


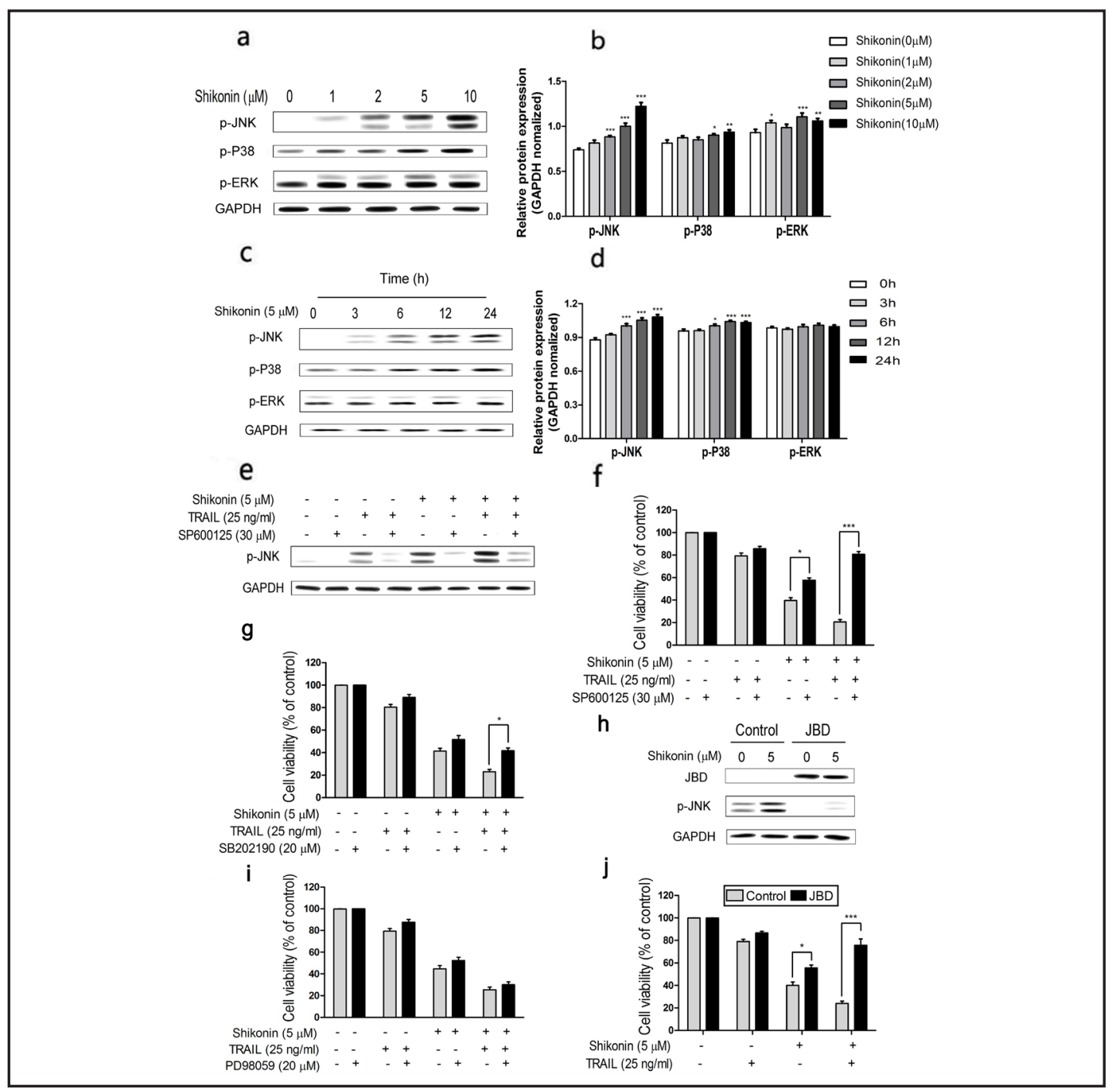

Fig. 2. JNK phosphorylation was essential for TRAIL enhanced anti-prolifration activity of shikonin in RBE CCA cells. (a, b) Western blotting analysis for protein expressions of p-JNK, p-P38 and p-ERK were measured in RBE cells treated with various concentration of shikonin. (c, d) Western blotting analysis for protein expressions of p-JNK, p-P38 and p-ERK were measured in RBE cells treated with indicated concentration of shikonin for different time. (e) Western blotting analysis for protein expression of p-JNK was measured in RBE cells treated with TRAIL and/or shkonin followed by the pretreatment of JNK inhibitor SP600125 for 2 h. (f, g, i) Cell viability was assessed after RBE cells were pretreated with JNK inhibitor SP600125, p38 inhibitor SB202190 or ERK inhibitor PD98059 for $2 \mathrm{~h}$ followed by treatment with TRAIL and/or shkonin. (h) Western blotting analysis for protein expressions of JBD and p-JNK were measured in RBE cells treated with shikonin and/or infected with JBD adenovirus vectors. (j) Cell viability was determined for RBE cells treated with TRAIL and/or shkonin followed by infection with JBD adenovirus vectors or empty vectors as control. Results are presented as the means \pm S.D. from at least 3 independent experiments. The statistical differences were calculated by the Student's t-test or one-way analysis of variance (ANOVA) with Dunnett's test. ${ }^{*} \mathrm{P}<0.05,{ }^{* *} \mathrm{P}<0.01,{ }^{* * *} \mathrm{P}<0.001$.

of cytochrome $C[29,30$.$] The dramatic decline of Bid protein expression was observed in$ accordance with shikonin and TRAIL induced caspase-8 activation, which was accompanied by the upregulation of Bax level in RBE cells (Fig. 1d and e). The further effect of TRAIL on shikonin cytotoxicity was elucidated using pan-caspase inhibitor (Q-VD-OPh) treated RBE 
a

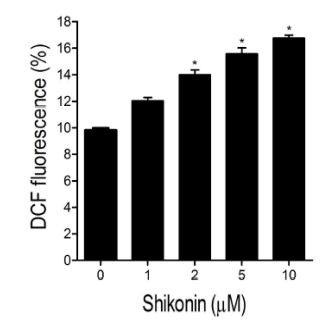

C

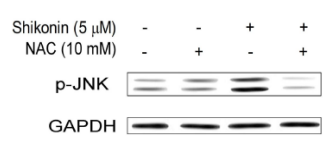

e

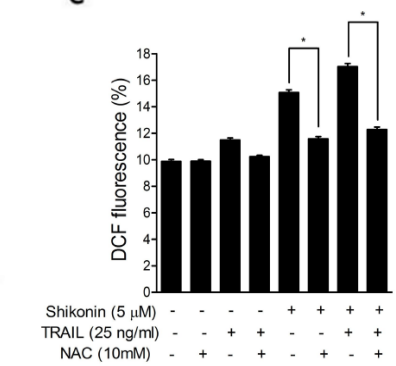

g

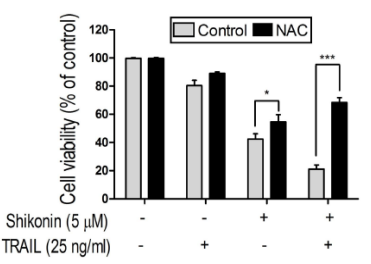

b

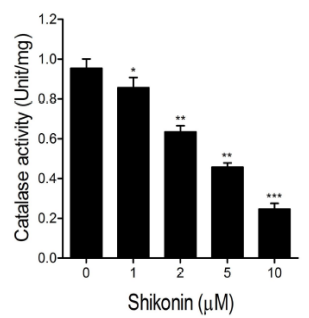

d

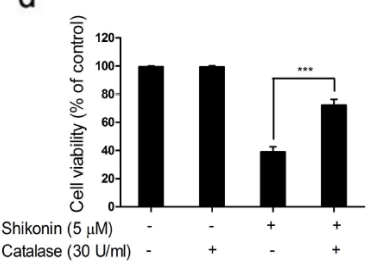

f

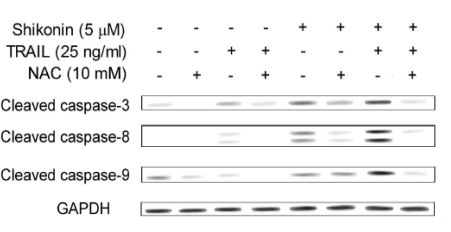

$\mathrm{h}$

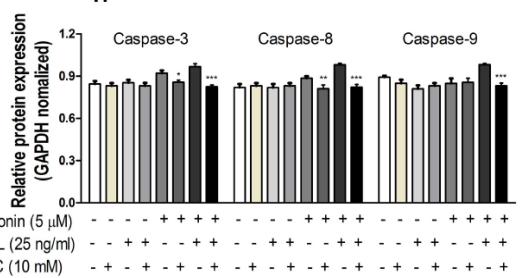

Fig. 3. Induction of ROS by shikonin leaded to TRAIL enhanced apoptosis. (a) Percentage of DCF fluorescence in RBE cells following treatment with DMSO or various concentrations of shikonin for $12 \mathrm{~h}$. (b) Catalase activity of RBE cells after treatment with various concentration of shikonin. (c) Western blotting analysis for protein expression of p-JNK was measured in RBE cells treated with shikonin and/or NAC. (d) Cell viability was assessed after RBE cells were incubated with catalase followed by treatment with TRAIL and/ or shkonin. (e) Percentage of DCF fluorescence in RBE cells treated with shikonin and/or TRAIL(f) Western blotting analysis for protein expressions of Cleaved caspase-3, 8, 9, were measured in RBE cells treated with TRAIL and/or shkonin followed by the pretreatment of NAC for $2 \mathrm{~h}$. (f, h) Western blotting analysis for protein expressions of Cleaved caspase-3, 8, 9, were measured in RBE cells treated with TRAIL and/or shkonin followed by the pretreatment of NAC for $2 \mathrm{~h}$. (g) Cell viability was assessed after RBE cells were pretreated with NAC for $2 \mathrm{~h}$ followed by treatment with TRAIL and/or shkonin. Results are presented as the means \pm S.D. from at least 3 independent experiments. The statistical differences were calculated by the Student's t-test or one-way analysis of variance (ANOVA) with Dunnett's test. ${ }^{*} \mathrm{P}<0.05,{ }^{* *} \mathrm{P}<0.01,{ }^{* * *} \mathrm{P}<0.001$.

cells. We found the caspase inhibitor significantly enhanced the cell viability in the group of shikonin and TRAIL treatment. However, the caspase inhibitor could not markedly influence the cell viability in TRAIL or shikonin alone treatment (Fig. 1h). These data suggested that TRAIL enhanced shikonin-induced apoptosis in RBE cells.

JNK activation was essential for TRAIL enhanced anti-proliferation activity of shikonin

To elucidate the molecular function of TRAIL enhanced anti-proliferation activity of shikonin in CCA cells, we first checked the protein expression levels of p-JNK, p-P38 and p-ERK 
Fig. 4. AKT activation had an effect on shikonin anti-proliferation activity. (a) Immunoblotting for p-AKT in RBE cells treated with indicated concentration of shikonin followed with or without pretreatment of AKT inhibitor LY for $4 \mathrm{~h}$. (b) Cell viability was assessed after RBE cells were pretreated with LY for 2 $\mathrm{h}$ followed by treatment with TRAIL and/or shkonin. (c) Immunoblotting for $\mathrm{p}$-AKT in RBE cells treated with indicated concentration of shikonin followed by transfection with Ad-AKT. (d) Cell viability was assessed after RBE cells were treatment with TRAIL and/or shkonin followed by transfection with

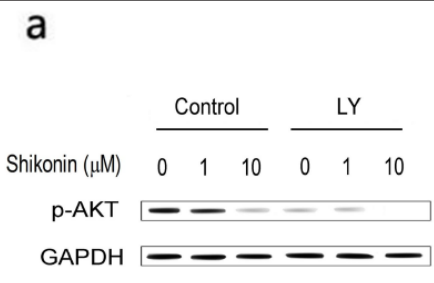

C

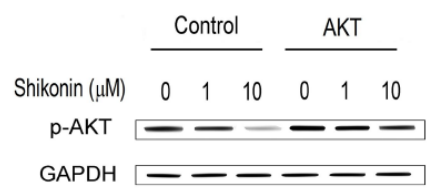

e

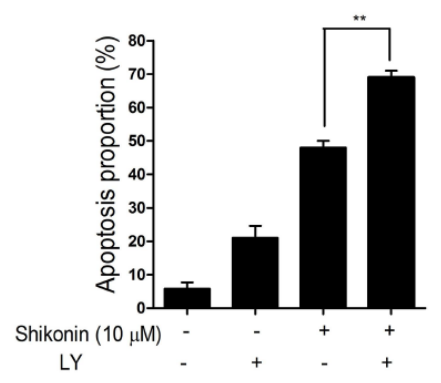

b

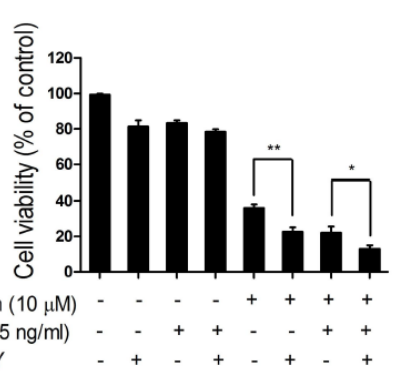

d

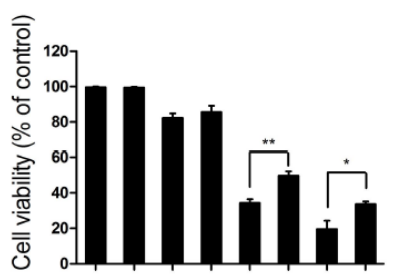

Shikonin $(10 \mu \mathrm{M})-$ - $^{2}++++$

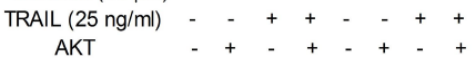

f

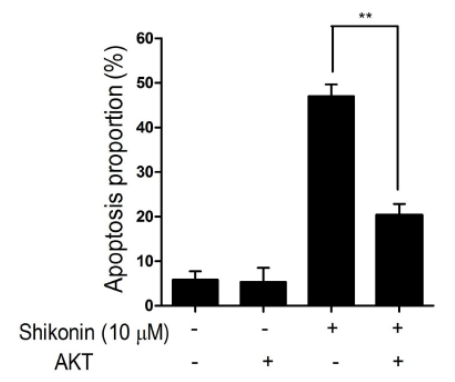

Ad-AKT. (e, f) Cells treated with indicated concentration of shikonin followed by transfection with Ad-AKT or pretreatment with LY for $2 \mathrm{~h}$. Cells were stained with annexin V-FITC/7-AAD and analyzed by flow cytometry. Results are presented as the means \pm S.D. from at least 3 independent experiments. The statistical differences were calculated by the Student's t-test or one-way analysis of variance (ANOVA) with Dunnett's test. ${ }^{*} \mathrm{P}<0.05,{ }^{* *} \mathrm{P}<0.01$.

by western blot assay with incubation of various concentration of shikonin. As indicated in Fig. 2a, the protein expression levels of p-JNK and p-P38 were obviously increased in a dosedependent manner. The relative protein expressions level of p-JNK, p-P38 and p-ERK were significantly increased compared with control (Fig. 2b). Furthermore, the protein expression levels of p-JNK and p-P38 were remarkably increased in a time-dependent manner, while not the p-ERK (Fig. 2c and d). To confirm the signaling cascades involved in TRAIL enhanced shikonin anti-proliferation activity, we next carried out experiments in the presence of SP600125 (JNK inhibitor), SB202190 (p38 MAPK inhibitor) and PD98059 (ERK inhibitor) on TRAIL and shikonin treatment cells. The p-JNK protein expression level was reduced by the JNK inhibitor incubation (Fig. 2e). As illustrated in Fig. 2f and g, SP600125 (30 $\mu \mathrm{M})$ or SB202190 $(20 \mu \mathrm{M})$ effectively enhanced the cell viability after cells treatment with TRAIL and shikonin, and SP600125 increased the cell viability in shikonin alone treatment. However, PD98059 $(20 \mu \mathrm{M})$ have not obviously enhanced the cell viability after cells treatment with TRAIL and shikonin (Fig. 2i). Consistently, overexpression of the JBD, a binding protein of JNK, could also efficiently suppress the increase protein level of p-JNK analyzed by western 
Fig. 5. Shikonin induced DR5 upregulation was essential for TRAIL enhanced apoptosis. (a) Real-time PCR analysis of DR5 mRNA from RBE cells treated with various concentration of shikonin for $24 \mathrm{~h}$. (b) Western blotting analysis for protein expressions of DR5 and cleaved caspase-3 were measured in RBE cells treated with TRAIL and/ or shkonin followed by the transfection with DR5 small interfering RNA (Si-DR5). (c) DR5 expression levels on the surface of RBE cells were determined using flow cytometry. (d) Western blotting analysis for protein expression of $\mathrm{p}$-JNK was measured in RBE cells treated with TRAIL and/or shkonin followed by the transfection with Si-DR5. (e) Percentage of DCF fluorescence in RBE cells following treatment with TRAIL and/or shkonin followed by the transfection with Si-DR5. (f) Western blotting analysis for protein expressions of DR5 was measured in RBE cells treated with shikonin and/or NAC. (g) Cell viability was assessed af-

a

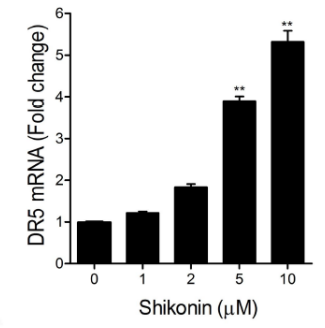

C

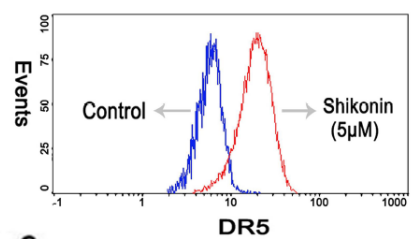

e
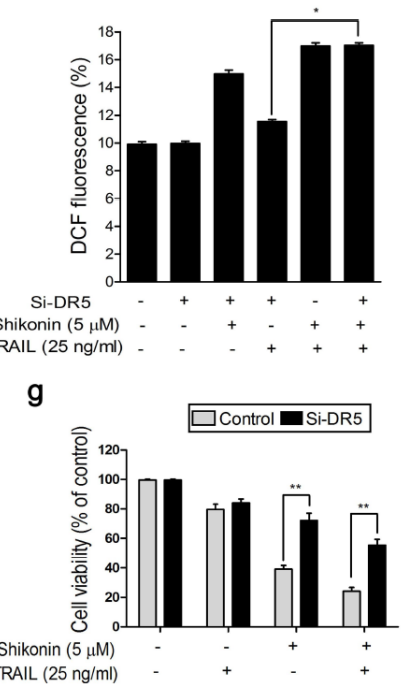

b

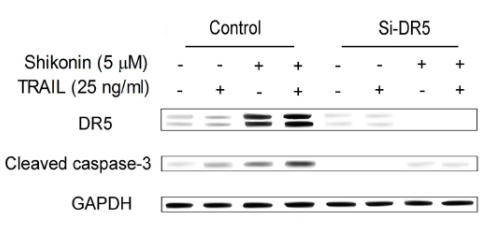

d

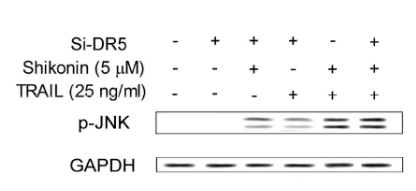

f

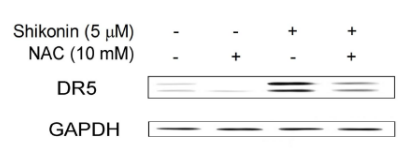

h

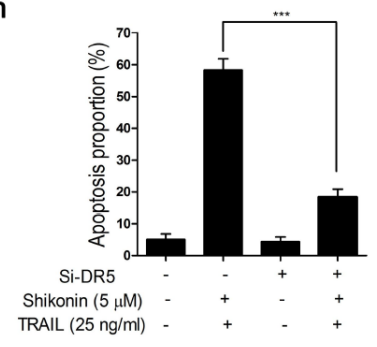

ter RBE cells were transfected with Si-DR5 followed by treatment with TRAIL and/or shkonin. (h) Cells were stained with annexin V-FITC/7-AAD and analyzed by flow cytometry. Results are presented as the means \pm S.D. from at least 3 independent experiments. The statistical differences were calculated by the Student's t-test or one-way analysis of variance (ANOVA) with Dunnett's test. ${ }^{*} \mathrm{P}<0.05,{ }^{* *} \mathrm{P}<0.01,{ }^{* * *} \mathrm{P}<0.001$.

blot with shikonin treatment in CCA cells (Fig. 2h). Overexpression of the JBD significantly enhanced cell viability in the group of TRAIL and shikonin treatment (Fig. 2j). These results suggested the activation of JNK pathway was required in TRAIL enhanced anti-proliferation activity of shikonin.

ROS triggered JNK was involved in TRAIL enhanced shikonin anti-proliferation activity and induced cell apoptosis

Previous studies have reported that shikonin could induced intracellular ROS generation $[31,32]$, and latest evidence suggested possible direct roles for ROS in regulating death receptor activation and apoptotic induction through the cluster of ROS-induced receptors and the formation of lipid raft-derived signaling platforms [33-35]. To determine whether the levels of intracellular ROS in shikonin-treated human CCA cells were altered and its effects on TRAIL enhanced shikonin anti-proliferation activity and induced cell apoptosis, we assessed intracellular ROS in human CCA cells by using the DCFH-DA (oxidation-sensitive fluorescent probe, 2',7'-dichlorofluorescein diacetate), which is based on the ROS-dependent oxidation 
of DCFH-DA to DCF (2'7'-dichlorofluorescein) [27]. As shown in Fig. 3a, the elevation of intracellular general ROS levels was observed in a dose-dependent manner. Next, RBE cells were incubated with or without shikonin following the treatment with $10 \mathrm{mM} \mathrm{NAC}$, a normal antioxidant and quite popular for their ability to minimize oxidative stress [36]. We found NAC significantly reversed shikonin-induced p-JNK protein expression (Fig. 3c). Consistently, NAC treatment significantly suppressed the apoptotic proteins level of cleaved caspase-3, -8 and -9 analyzed in CCA cells by blocking ROS production (Fig. $3 \mathrm{f}$ and $\mathrm{h}$ ). Moreover, NAC treatment remarkably decreased the ROS levels in CCA cells followed treatment with shikonin alone or shkonin and TRAIL (Fig. 3e). In addition, we detected the catalase activity and found it was obviously decreased with the increasing concentration of shikonin treatment (Fig. 3b). The anti-proliferation activity of shikonin was markedly attenuated by catalase (Fig. 3d) or NAC treatment in the presence of TRAIL (Fig. 3g). These results indicated ROS triggered JNK was involved in TRAIL enhanced shikonin anti-proliferation activity and induced cell apoptosis.

AKT activation had an effect on anti-proliferation activity of shikonin

To determine whether AKT was involved in the regulation of shikonin-induced cell death in the presence of TRAIL, we analyzed the effect of shikonin on AKT activation. Treatment of RBE cells with shikonin showed reduction in AKT activation, especially in the high concentration of shikonin treatment. Exposure of RBE cells to shikonin, followed by LY29400, a PI3K-AKT pathway inhibitor, down-regulated the protein expression level of p-AKT (Fig. 4a). However, treatment with LY29400 enhanced the anti-proliferation activity and apoptosis proportion of shikonin (Fig. 4b and e), the activity of shikonin in cells was not changed with or without TRAIL treatments (Fig. 4b). Moreover, overexpression with AKT up-regulated the protein expression level of p-AKT (Fig. 4c). The anti-proliferation activity and apoptosis proportion of shikonin were reduced after transfection with Ad-AKT (Fig. $4 \mathrm{~d}$ and f), while the activity of shikonin in cells was not changed with or without TRAIL treatments (Fig. 4d). These findings showed that AKT activation had an effect on shikonin anti-proliferation activity, but not in the TRAIL enhanced counterparts.

Shikonin induced DR5 upregulation and contributed to TRAIL enhanced apoptosis

DR5 was known for the regulation of TRAIL induced apoptosis. Thus, we investigated whether DR5 potentiated TRAIL enhanced apoptosis of shikonin. Treatment of RBE cells with shikonin led to an increased DR5 mRNA expression in a dose-dependent manner (Fig. 5 a), and obviously elevated protein expression level in shikonin treatment compared with DMSO group (Fig. 5b). Furthermore, shikonin increased DR5 expression level on the surface of RBE cells (Fig. 5c). DR5 small interfering RNA (Si-DR5) abrogated the effects of TRAIL on shikonin induced anti-proliferation activity and apoptosis proportion in RBE cells (Fig $5 \mathrm{~g}$ and $\mathrm{h}$ ). Transfection of RBE cells with Si-DR5 markedly reduced shikonin-induced DR5 protein expression level (Fig. 5b). Moreover, cleaved caspase-3 protein expression level induced by shikonin plus TRAIL treatment was significantly attenuated in cells transfected with Si-DR5 (Fig. 5b). However, transfection with Si-DR5 has not altered the JNK expression with the shikonin and/or TRAIL treatment, as well as ROS level (Fig. 5d and e). However, NAC treatment followed shikonin obviously reduced the DR5 expression level (Fig. 5f). The anti-proliferation of shikonin was also markedly decreased after Si-DR5 transfection in the presence of TRAIL (Fig. 5g). However, cells transfected with Si-DR5 and treated with TRAIL or shikonin were not significantly changed for the cytotoxicity (Fig. 5g). In addition, we also examined whether the silencing of DR5 could reverse the effects of TRAIL on shikonin-induced cell apoptosis. The results showed that cell apoptosis induced by TRAIL plus shikonin treatment was remarkably inhibited by Si-DR5 transfection (Fig. 5h). Taken together, these results suggested that shikonin-induced DR5 up-regulation was essential for TRAIL-enhanced effects of shikonin in RBE cells. 


\section{Cellular Physiology Cell Physiol Biochem 2017;42:1073-1086 \\ \begin{tabular}{c|c|c|} 
DOI: 10.1159/000478758 & and Biochemistry Published online: June 29, 2017 & $\begin{array}{l}\text { O 2017 The Author(s). Published by S. Karger AG, Basel } \\
\text { www.karger.com/cpb }\end{array}$
\end{tabular}

Zhou et al.: Mechanism Role of Shikonin in Cholangiocarcinoma

\section{Discussion}

Reactive oxygen species (ROS) is a heterogeneous group of diatomic oxygen from free or non-free radical species. The most common source of ROS is from mitochondria which serve as the center of ATP synthesis. ROS play a pivotal role in the pathogenesis of various human diseases, including cancer. By causing DNA mutations, regulating gene expression, influencing various signaling pathways, ROS widely participates in carcinogensis [37]. Increasing evidence suggests that ROS may serve as a tumor promoter that initiate carcinogensis, by inducing cell proliferation, survival and cellular migration. However, ROS is also known as a tumor suppressor in numerous studies [38]. This chemically highly reactive ROS promotes oxidative stress and contributes to cell damage and even death [39]. Therefore, a number of anti-tumor therapeutic approaches are designed via ROS-mediated mechanism. For instance, surfactin induced cell apoptosis by activating ROS. The dramatic up-regulation of ROS activated MAPK subfamilies and led to the apoptosis of breast cancer cells [40]. Diaporine induced the apoptosis of breast cancer cells through increasing ROS release [41]. In prostate cancer, hydroxychavicol-induced ROS triggered mitochondriallymediated intrinsic apoptosis [42]. Zhong et al. demonstrated that guggulsterone-induced apoptosis of CCA cells was achieved by ROS mediated JNK signaling pathway in CCA [26].

The interaction between shikonin and reactive oxygen species (ROS) was first established by Gao et al. [43]. Shikonin was demonstrated to induce apoptosis in hepatoma cells through an ROS-mediated oxidative stress pathway [44]. In this study, we found that shikonin could inhibit cell viability and induce apoptosis of CCA cells, effects enhanced by TRAIL treatment via activation of caspase- $3,-8,-9$. The apoptosis pathway could be activated by combination of TRAIL and shikonin through up-regulation of ROS production. Since ROS has been well considered as a key mediator in many signaling pathways, including MAPK and AKT signal transduction cascades, western blot analysis and flow cytometry analysis were performed to measure the effect of ROS on signaling pathways. The up-regulation of p-JNK, p-P38 and the down-regulation of p-AKT occurred in a shikonin treatment group. We found inhibition of AKT markedly enhanced anti-proliferation activity of shikonin in shikonin treatment group, while AKT overexpression could markedly restore TRAIL enhanced cell death by shikonin treatment. We hypothesized that AKT inactivation was involved in TRAIL enhanced cell death by shikonin. However, the relationship between MAPK and AKT signaling in TRAIL enhanced cell death by shikonin need further study. We found cell viability was significantly enhanced and caspase- $3,-8,-9$ protein expression levels were markedly attenuated after NAC treatment in combination of TRAIL and shikonin group, while shikonin alone treatment obtained less effects. These results further demonstrated that TRAIL enhanced apoptosis via shikonin induced ROS. In further, we also found SP600125 treatment or overexpression of the JBD could remarkably promote the cell viability in the group of TARIL plus shikonin. In addition, NAC treatment could significantly reverse shikonin-induced p-JNK protein expression, which indicated that shikonin induced CCA apoptosis via ROS mediated JNK signalling. These results suggested activation of JNK by ROS mediation is required in TRAIL

Fig. 6. A diagram of the pathways in which TRAIL enhances shikonin induced apoptosis in cholangiocarcinoma cells. Green represent activation, red represent inhibition.

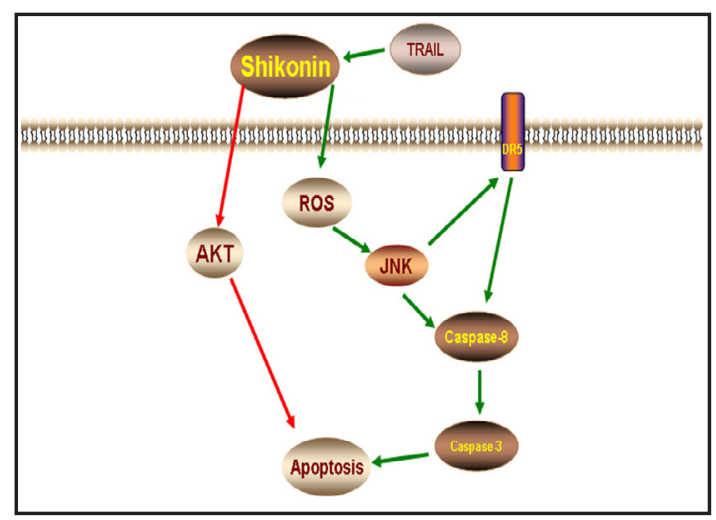


Zhou et al.: Mechanism Role of Shikonin in Cholangiocarcinoma

enhanced anti-proliferation activity of shikonin. Finally, caspase-3 protein expression and apoptosis induced by shikonin plus TRAIL were significantly attenuated after cells transfected with Si-DR5, and cell viability in same treatment could significantly promote compared with control. Previous study demonstrated that DR5 could induce JNK activation in colon cells [45], whereas most of other studies showed that ROS/JNK signalling mediated DR5 in various cell lines [46, 47], which were consistent with our results. In addition, we also found that Si-DR5 transfection has no effect on shikonin induced cell death. This findings were similar to previous study [47]. These results suggested that shikonin-induced DR5 upregulation was essential for TRAIL-enhanced effects of shikonin in RBE cells.

In summary, we concluded that we found shikonin could inhibit cell viability and induce apoptosis of CCA cells, effects enhanced by TRAIL treatment via ROS mediated JNK signalling pathways, involvement in up-regulation of DR5 expression (Fig. 6). Our results provided a further understanding of the mechanism underlying the anti-tumor effects of shikonin by TRAIL enhanced in CCA and a new therapeutic strategy to CCA treatment.

\section{Acknowledgements}

This work was supported by grants from National Nature and Science Foundation of China (NSFC) (81470856 to YZ, 81001324 and 81373163 to RT), Zhejiang Provincial Nature and Science Foundation (NSF) grant for "Outstanding Youth" (No. LR15H100001 to RT).

\section{Disclosure Statement}

The authors declare no competing interests.

\section{References}

Gores GJ: Cholangiocarcinoma: Current concepts and insights. Hepatology 2003;37:961-969.

Ghouri YA, Mian I, Blechacz B: Cancer review: Cholangiocarcinoma. J Carcinog 2015;14:1.

Blechacz B, Komuta M, Roskams T, Gores GJ: Clinical diagnosis and staging of cholangiocarcinoma. Nat Rev Gastroenterol Hepatol 2011;8:512-522.

-4 Elserag HB: Risk factors for cholangiocarcinoma. Hepatology 2011;54:173-184.

5 Shin H, Oh J, Masuyer E, Curado M, Bouvard V, Fang Y, Wiangnon S, Sripa B, Hong S: Epidemiology of cholangiocarcinoma: An update focusing on risk factors. Cancer Sci 2010;101:579-585.

6 Rizvi S, Patel T, Gores GJ: Cholangiocarcinoma: Molecular pathways and therapeutic opportunities. Semin Liver Dis 2014;34:456-464.

7 Chen X, Yang L, Oppenheim JJ, Howard OMZ: Cellular pharmacology studies of shikonin derivatives. Phytother Res 2002;16:199-209.

-8 Singh B, Sharma MK, Meghwal PR, Sahu PM: Anti-inflammatory activity of shikonin derivatives from arnebia hispidissima. Phytomedicine 2003;10:375-380.

-9 Jeung YJ, Kim HG, Ahn J, Lee HJ, Lee SB, Won M, Jung CR, Im JY, Kim BK, Park SK, Son MJ, Chung KS: Shikonin induces apoptosis of lung cancer cells via activation of foxo3a/egr1/sirt1 signaling antagonized by p300. Biochim Biophys Acta 2016;1863:2584-2593.

10 He G, He G, Zhou R, Pi Z, Zhu T, Jiang L: Enhancement of cisplatin-induced colon cancer cells apoptosis by shikonin, a natural inducer of ros in vitro and in vivo. Biochem Biophys Res Commun 2016;469:1075-1082.

11 Wei Y, Li M, Cui S, Wang D, Zhang C, Zen K, Li L: Shikonin inhibits the proliferation of human breast cancer cells by reducing tumor-derived exosomes. Molecules 2016;21:777.

-12 Deng B, Feng Y, Deng B: TIPE2 Mediates the Suppressive Effects of Shikonin on MMP13 in Osteosarcoma Cells. Cell Physiol Biochem 2015;37:2434-2443.

13 Trivedi R, Müller GA, Rathore MS, Mishra DP, Dihazi H: Anti-Leukemic Activity of Shikonin: Role of ERP57 in Shikonin Induced Apoptosis in Acute Myeloid Leukemia. Cell Physiol Biochem 2016;39:604-616. 


\section{Cellular Physiology Cell Physiol Biochem 2017;42:1073-1086

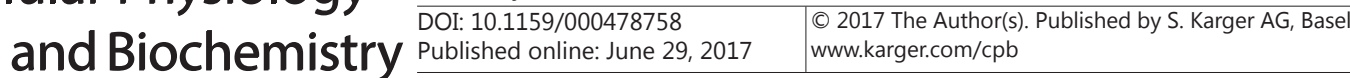

Zhou et al.: Mechanism Role of Shikonin in Cholangiocarcinoma

14 Chen Y, Zheng L, Liu J, Zhou Z, Cao X, Lv X, Chen F: Shikonin inhibits prostate cancer cells metastasis by reducing matrix metalloproteinase-2/-9 expression via akt/mtor and ros/erk1/2 pathways. Int Immunopharmaco 2014;21:447-455.

15 Deng B, Qiu B: Shikonin inhibits invasiveness of osteosarcoma through mmp13 suppression. Tumor Biol 2015;36:9311-9317.

-16 Jang SY, Lee JK, Jang EH, Jeong SY, Kim J: Shikonin blocks migration and invasion of human breast cancer cells through inhibition of matrix metalloproteinase-9 activation. Oncol Rep 2014;31:2827-2833.

-17 Hong D, Jang SY, Jang EH, Jung B, Cho I, Park M, Jeong SY, Kim J: Shikonin as an inhibitor of the lps-induced epithelial-to-mesenchymal transition in human breast cancer cells. Int J Mol Med 2015;36:1601-1606.

18 Gara RK, Srivastava VK, Duggal S, Bagga JK, Bhatt M, Sanyal S, Mishra DP: Shikonin selectively induces apoptosis in human prostate cancer cells through the endoplasmic reticulum stress and mitochondrial apoptotic pathway. J Biomed Sci 2015;22:26-26.

19 Tian R, Li Y, Gao M: Shikonin causes cell-cycle arrest and induces apoptosis by regulating the egfr-nf-кb signalling pathway in human epidermoid carcinoma a431 cells. Biosci Rep 2015;35: e00189.

20 Jang SY, Hong D, Jeong SY, Kim J: Shikonin causes apoptosis by up-regulating p73 and down-regulating icbp90 in human cancer cells. Biochem Biophys Res Commun 2015;465:71-76.

21 Yeh Y, Liu T, Lai H: Shikonin induces apoptosis, necrosis, and premature senescence of human a549 lung cancer cells through upregulation of p53 expression. Evid-based Compl Alt 2015;2015: 620383.

-22 Ko H, Kim SJ, Shim SH, Chang H, Ha CH: Shikonin induces the apoptotic cell death via regulation of p53 and nrf2 in ags human stomach carcinoma cells. Biomol Ther 2016;24:501-509.

23 Pan G, Ni J, Wei YF, Yu G, Gentz R, Dixit VM: An antagonist decoy receptor and a death domain-containing receptor for TRAIL. Science 1997;277:815-818.

-24 Sriraksa R, Limpaiboon T: TRAIL in Combination with Subtoxic 5-FU Effectively Inhibit Cell Proliferation and Induce Apoptosis in Cholangiocarcinoma Cells. Asian Pac J Cancer P 2015;16:6991-6996.

-25 Panichakul T, Intachote P, Wongkajorsilp A, Sripa B, Sirisinha S: Triptolide sensitizes resistant cholangiocarcinoma cells to TRAIL-induced apoptosis. Anticancer Res 2006;26:259-265.

26 Zhong F, Tong Z, Fan L, Zha L, Wang F, Yao M, Gu K: Guggulsterone-induced apoptosis in cholangiocarcinoma cells through ros/jnk signaling pathway. Am J Cancer Res 2016;6:226-237.

27 Shin YJ, Hyon JY, Kim S, Koh JW, Kwon SI, Wee WR: Cysteamine suppresses human peripheral blood mononuclear cells--human corneal endothelial cell reaction via reactive oxygen species reduction. Mol Vis 2011;17:3371-3378.

28 Wang H, Joseph JA: Quantifying cellular oxidative stress by dichlorofluorescein assay using microplate reader. Free Radic Biol Med 1999;27:612-616.

29 Li H, Zhu H, Xu CJ, Yuan J: Cleavage of bid by caspase 8 mediates the mitochondrial damage in the fas pathway of apoptosis. Cell 1998;94:491-501.

-30 Desagher S, Osen-Sand A, Nichols A, Eskes R, Montessuit S, Lauper S, Maundrell K, Antonsson B, Martinou JC: Bid-induced conformational change of bax is responsible for mitochondrial cytochrome c release during apoptosis. J Cell Biol 1999;144:891-901.

-31 Chen CH, Chern CL, Lin CC, Lu FJ, Shih MK, Hsieh PY, Liu TZ: Involvement of reactive oxygen species, but not mitochondrial permeability transition in the apoptotic induction of human sk-hep-1 hepatoma cells by shikonin. Planta Med 2003;69:1119-1124.

-32 Mao X, Yu CR, Li WH, Li WX: Induction of apoptosis by shikonin through a ros/jnk-mediated process in bcr/ abl-positive chronic myelogenous leukemia (cml) cells. Cell Res 2008;18:879-888.

33 Zhang AY, Yi F, Zhang G, Gulbins E, Li PL: Lipid raft clustering and redox signaling platform formation in coronary arterial endothelial cells. Hypertension 2006;47:74-80.

-34 Zhang AY, Yi F, Jin S, Xia M, Chen QZ, Gulbins E, Li PL: Acid sphingomyelinase and its redox amplification in formation of lipid raft redox signaling platforms in endothelial cells. Antioxid Redox Sign 2007;9:817-828.

35 Circu ML, Aw TY: Reactive oxygen species, cellular redox systems, and apoptosis. Free Radic Biol Med 2010;48:749-762.

-36 Kerksick C, Willoughby D: The antioxidant role of glutathione and n-acetyl-cysteine supplements and exercise-induced oxidative stress. J Int Soc Sport Nutr 2005;2:38-44.

-37 Klaunig JE, Kamendulis LM: The role of oxidative stress in carcinogenesis. Annu Rev Pharmacol Toxicol 2004;44:239-267.

-38 Storz PD: Reactive oxygen species in tumor progression. Front Biosci 2005;10:1881-1896. 


\section{Cellular Physiology Cell Physiol Biochem 2017;42:1073-1086 \begin{tabular}{l|l} 
DOI: 10.1159/000478758 \\
and Biochemistry
\end{tabular} \begin{tabular}{l}
$\begin{array}{l}\text { O 2017 The Author(s). Published by S. Karger AG, Basel } \\
\text { www.karger.com/cpb }\end{array}$ \\
\hline
\end{tabular}}

Zhou et al.: Mechanism Role of Shikonin in Cholangiocarcinoma

39 Droge W: Free radicals in the physiological control of cell function. Physiol Rev 2002;82:47-95.

40 Cao X, Wang A, Wang C, Lu M, Cui Y, Jiao R: Surfactin induces apoptosis in human breast cancer mcf-7 cells through a ros/jnk-mediated mitochondrial/caspase pathway. Chem Biol Interact 2010;183:357-362.

41 Feng X, Yu W, Zhou F, Chen J, Shen P: A novel small molecule compound diaporine inhibits breast cancer cell proliferation via promoting ros generation. Biomed Pharmacother 2016;83:1038-1047.

42 Gundala SR, Yang C, Mukkavilli R, Paranjpe R, Brahmbhatt M, Pannu V, Cheng A, Aneja R: Hydroxychavicol, a betel leaf component, inhibits prostate cancer through ros-driven DNA damage and apoptosis. Toxicol Appl Pharmacol 2014;280:86-96.

-43 Gao D, Kakuma M, Oka S, Sugino K, Sakurai H: Reaction of $\beta$-alkannin (shikonin) with reactive oxygen species: Detection of $\beta$-alkannin free radicals. Bioorg Med Chem 2000;8:2561-2569.

-44 Chen C, Chern C, Fungjou LU, Shih M, Hsieh P, Liu T: Involvement of reactive oxygen species, but not mitochondrial permeability transition in the apoptotic induction of human sk-hep-1 hepatoma cells by shikonin. Planta Med 2003;69:1119.

45 Mahalingam D, Keane M, Pirianov G, Mehmet H, Samali A, Szegezdi E: Differential activation of JNK1 isoforms by TRAIL receptors modulate apoptosis of colon cancer cell lines. Br J Cancer 2009; 100:14151424.

-46 Sung B, Prasad S, Ravindran J, Yadav VR, Aggarwal BB: Capsazepine, a TRPV1 antagonist, sensitizes colorectal cancer cells to apoptosis by TRAIL through ROS-JNK-CHOP-mediated upregulation of death receptors. Free Radic Biol Med 2012;53:1977-1987.

47 Tse AK, Cao HH, Cheng CY, Kwan HY, Yu H, Fong WF, Yu ZL: Indomethacin Sensitizes TRAIL-Resistant Melanoma Cells to TRAIL-Induced Apoptosis through ROS-Mediated Upregulation of Death Receptor 5 and Downregulation of Survivin. J Invest Dermatol 2014;134: 1397-1407. 